\title{
TUBEROUS SCLEROSIS: A CASE REPORT
}

\author{
Vaddadi Srinivas ${ }^{1}$, D. Markendeyulu ${ }^{2}$, L. Sandhyarani ${ }^{3}$, Leena Mehenaz ${ }^{4}$, A. S. V. Kartheek ${ }^{5}$
}

${ }_{1}^{1}$ Professor, Department of Medicine, Rangaraya Medical College, Kakinada.

${ }^{2}$ Assistant Professor, Department of Medicine, Rangaraya Medical College, Kakinada.

${ }^{3}$ Assistant Professor, Department of Medicine, Rangaraya Medical College, Kakinada.

${ }^{4}$ Post Graduate, Rangaraya Medical College, Kakinada.

${ }^{5}$ Post Graduate, Rangaraya Medical College, Kakinada.

\section{ABSTRACT}

We present a case of young patient who came to the hospital with tonic-clonic seizures, on evaluation found to have neurocutaneous markers and various radiological manifestations, thus diagnosed as Tuberous Sclerosis.

\section{KEYWORDS}

Hamartin, Tuberin, Angiofibromas, Shagreen Patch.

HOW TO CITE THIS ARTICLE: Srinivas V, Markendeyulu D, Sandhyarani L, et al. Tuberous sclerosis: a case report. J Evolution Med Dent Sci 2016;5(1):96-99, DOI: 10.14260/jemds/2016/22

\section{INTRODUCTION}

Tuberous Sclerosis or Tuberous Sclerosis Complex is a genetic disorder characterized by the growth of numerous benign tumors in many parts of the body including the brain, heart, lungs, eyes, kidneys, skin and other organs, leading to seizures, intellectual disability, autism or developmental delay. TSC is caused by mutations on either of two genes, TSC1 (Long arm of chromosome 9) and TSC2 (Short arm of chromosome 16), which encode for the proteins hamartin and tuberin respectively.[1] These proteins act as tumor growth suppressors, agents that regulate cell proliferation and differentiation. A 1998 study estimated total population prevalence between about 7 and 12 cases per 100 live births. TSC occurs in all races and ethnic groups, and in both genders. The cellular elements within the nodular cerebral regions called tubers are abnormal in number and size. The tumor like growths in different organs may include different cell types (ex: fibroblasts, angioblasts, glioblasts, neuroblasts and cardiac myoblasts). These cells proliferate during embryonic development and turn into malignant transformation at later years.

Tuberous sclerosis complex presents with variable clinical manifestations together with angiofibromas distributed in a characteristic "butterfly" pattern on the face and forehead. The most important neurological problems are mental retardation, seizures, and autism and learning difficulties. The diagnostic criteria of TSC have been divided into major and minor features. ${ }^{[2]}$

A possible diagnosis of tuberous sclerosis will be made when an individual has either: 1 major feature or 1 major and 1 minor feature or more than 2 minor.

Financial or Other, Competing Interest: None.

Submission 13-12-2015, Peer Review 14-12-2015

Acceptance 30-12-2015, Published 04-01-2016.

Corresponding Author:

Dr. Vaddadi Srinivas,

FICP,

Professor of Medicine

Rangaraya Medical College,

Kakinada.

E-mail: drvaddadisrinivas@gmail.com

DOI:10.14260/jemds/2016/22

\section{Major Criteria}

Angiofibromas (3 or more) or forehead plaque, Hypomelanotic macules ( 3 or more), Ungual fibromas (2 or more), Shagreen patch or multiple collagenomas, Multiple retinal hamartomas, Cortical dysplasia's (More than 3). This includes tubers and cerebral white matter radial migration lines. Subependymal nodule(s), Subependymal giant cell astrocytoma (s), Cardiac rhabdomyoma, Lymphangioleiomyomatosis (LAM), Angiomyolipomas (2 or more).

\section{Minor Criteria}

Multiple randomly distributed pits in dental enamel, Hamartomatous Rectal Polyps, Bone cysts, cerebral white matter migration tracts, gingival fibromas, Non-renal hamartomas, Retinal achromic patches, Confetti skin lesions, multiple renal cysts.

\section{CASE}

A 35-year-old patient, farmer by occupation came with complaints of 2 episodes of seizures of 1-day duration. Each episode lasted for 1-2 minutes with tonic clonic activity involving all the four limbs with associated loss of consciousness, frothing from the mouth, up-rolling of eye balls and involuntary micturition. These two episodes of seizures occurred within an interval of 15 minutes with no regain of consciousness. There was no history of trauma, fever, headache, vomiting or weakness of limbs. Patient had a past history of convulsions since 25 years of age, he was on antiepileptic drugs but the compliance is not maintained. There was no history of delayed milestones but his performance at school was poor. He was married and has eight years old child who has no history of seizures. He was born to nonconsanguineous parents but has an elder brother who suffered from seizures.

On general examination patient was conscious, coherent, no pallor, cyanosis or clubbing, but patient has multiple small angiofibromas (Adenoma sebaceum) present on either side of nose in a butterfly fashion [Fig 1]. A $7 \times 2 \mathrm{~cm}$ hypo-pigmented macule present on left arm suggestive of ash leaf macule [Fig 2], Shagreen patch on right lower back [Fig 3]. Subungual fibromas were seen on left thumb with dystrophic nail changes [Fig 4]. His vitals were pulse $88 / \mathrm{min}$, blood pressure $110 / 70 \mathrm{mmHg}$, respiratory rate $16 / \mathrm{min}$ and temperature normal. 
CNS Examination showed his higher mental functions were normal, speech normal and his mini mental score examination (MMSE-14/30), cranial nerve, fundus examination normal. His motor, sensory, cerebellar systems were normal, there was no evidence of involuntary movements or meningeal irritation signs, spine and cranium were normal.

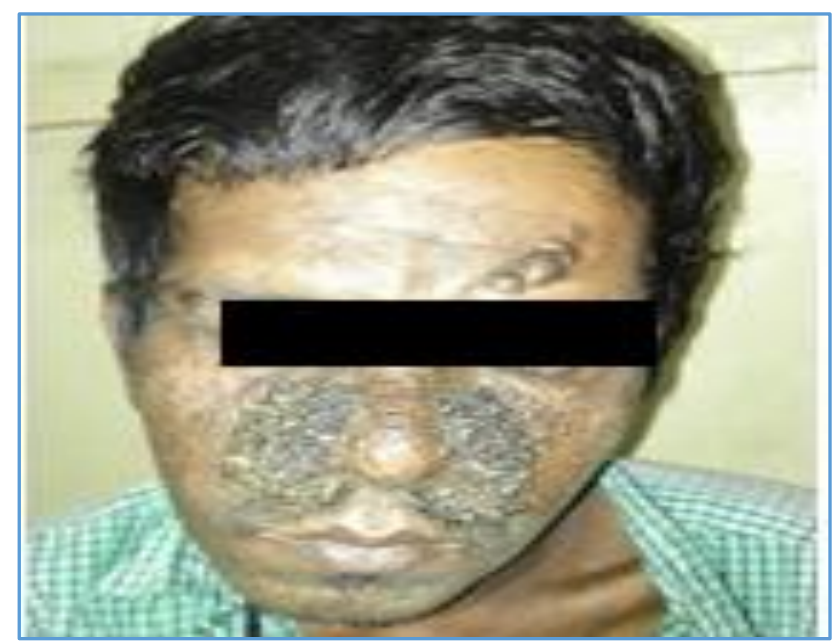

Fig. 1: Butterfly Angiofibromas [Adenoma Sebaceum]

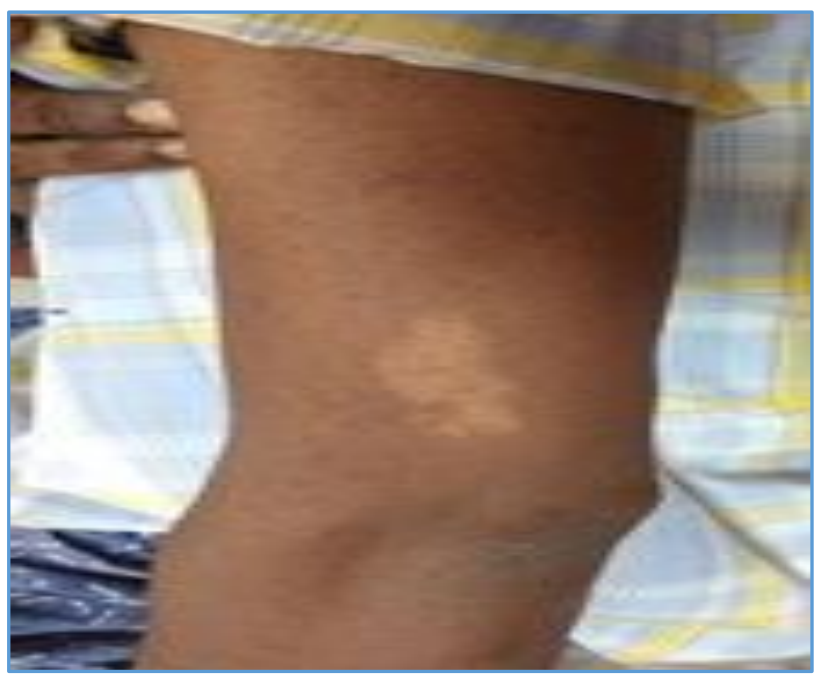

Fig. 2: Hypo-Melanotic Ash Leaf Macule

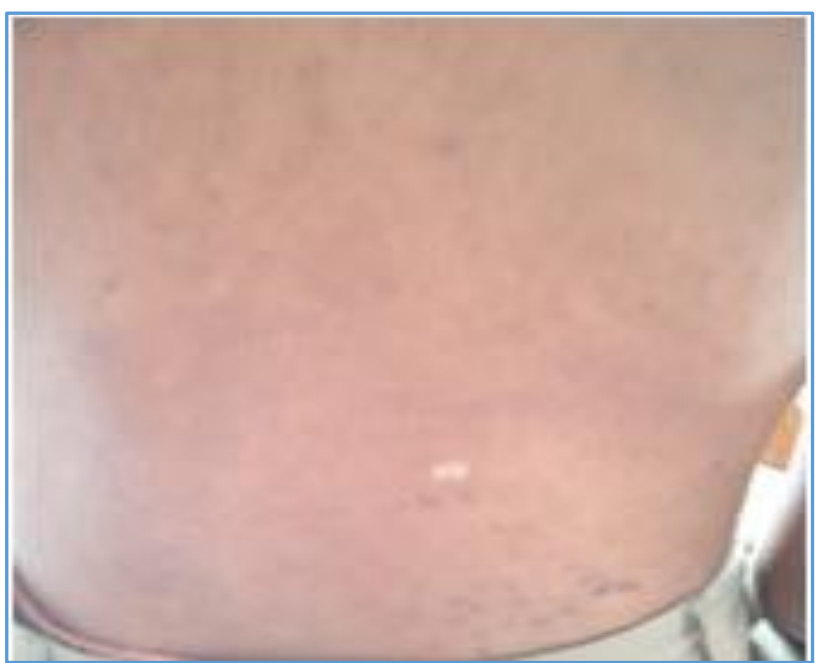

Fig. 3: Shagreen patches on Lower back

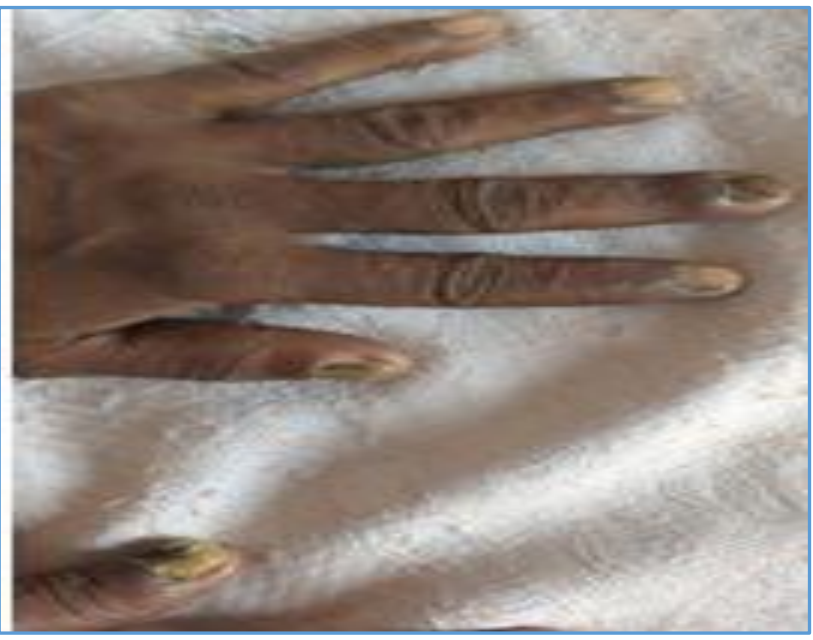

Fig. 4: Subungual Fibromas on Nails

Lab investigation revealed hemoglobin $8 \mathrm{gm} \%$, total leukocyte count 7100/cumm, differential count P56\% L40\% E4\%, ESR $40 \mathrm{~mm} / 1 \mathrm{hr}$, Blood sugar $90 \mathrm{mg} / \mathrm{dl}$, Urea $22 \mathrm{mg} / \mathrm{dl}$, Creatinine $0.5 \mathrm{mg} / \mathrm{dl}$, urine examination normal. X-ray both hands, X-ray chest and electrocardiogram were normal. Echocardiogram findings were not suggestive of any chamber enlargement, RWMA or any mass lesions and ejection fraction $70 \%$.

Ultrasonogram of abdomen showed irregular hyperechoic vascular lesion measuring $22 \times 20 \mathrm{~mm}$ size suggestive of angiomyolipoma in the mid pole of the kidney [Fig 5]. MRI scan brain revealed radial bands in bilateral frontal and posterior parietal regions, multiple focal cortical tubers diffusely involving cerebral hemisphere and right cerebellum, subependymal calcified nodules around both lateral ventricles and right foramen of Monro [Fig 6]. These features were suggestive of tuberous sclerosis. CT scan brain plain showed multiple calcifications seen in both subependymal and peri-ventricular regions called as bone stones suggestive of tuberous sclerosis [Fig 7].

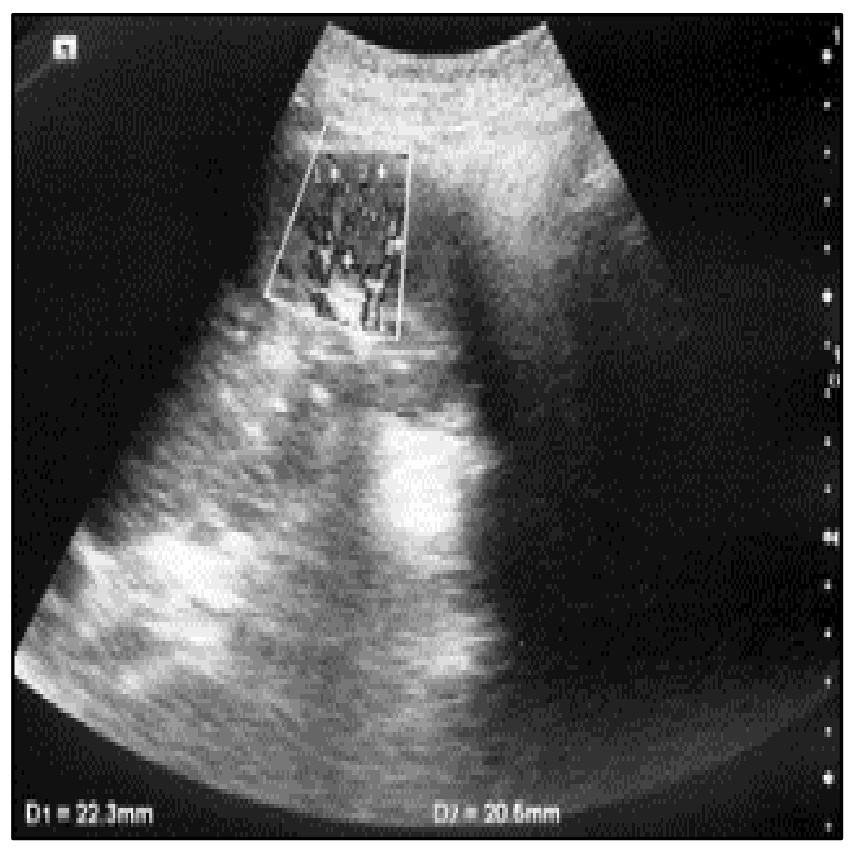

Fig. 5: US abdomen showing Hyperechoic Lesion (Angiomyolipoma) in Kidney 


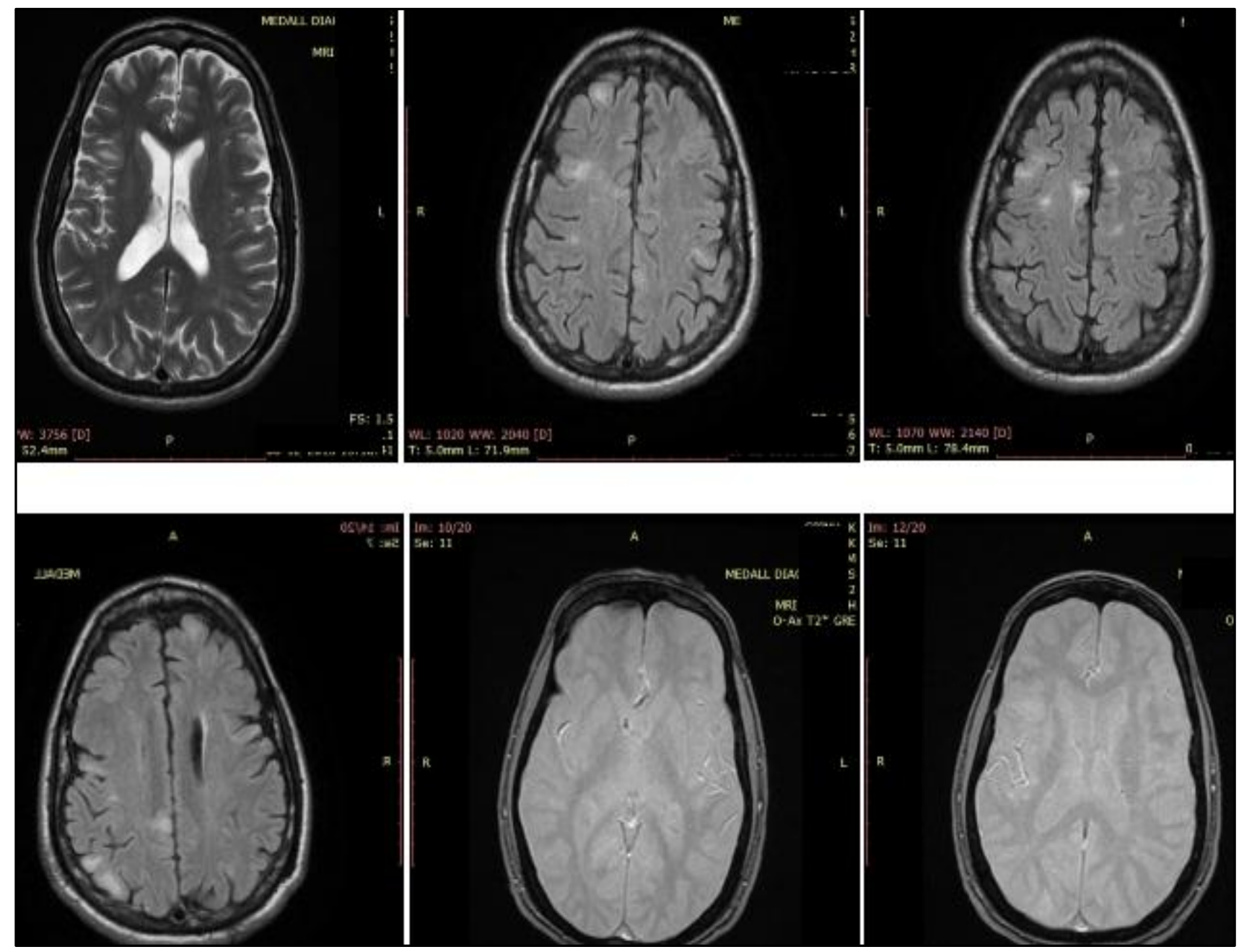

Fig. 6: MRI brain showing radial bands in bilateral frontal and posterior parietal regions, multiple cortical tubers in cerebral hemispheres and right cerebellum, subependymal calcified nodules around both Lateral Ventricles and Foramen of Monro

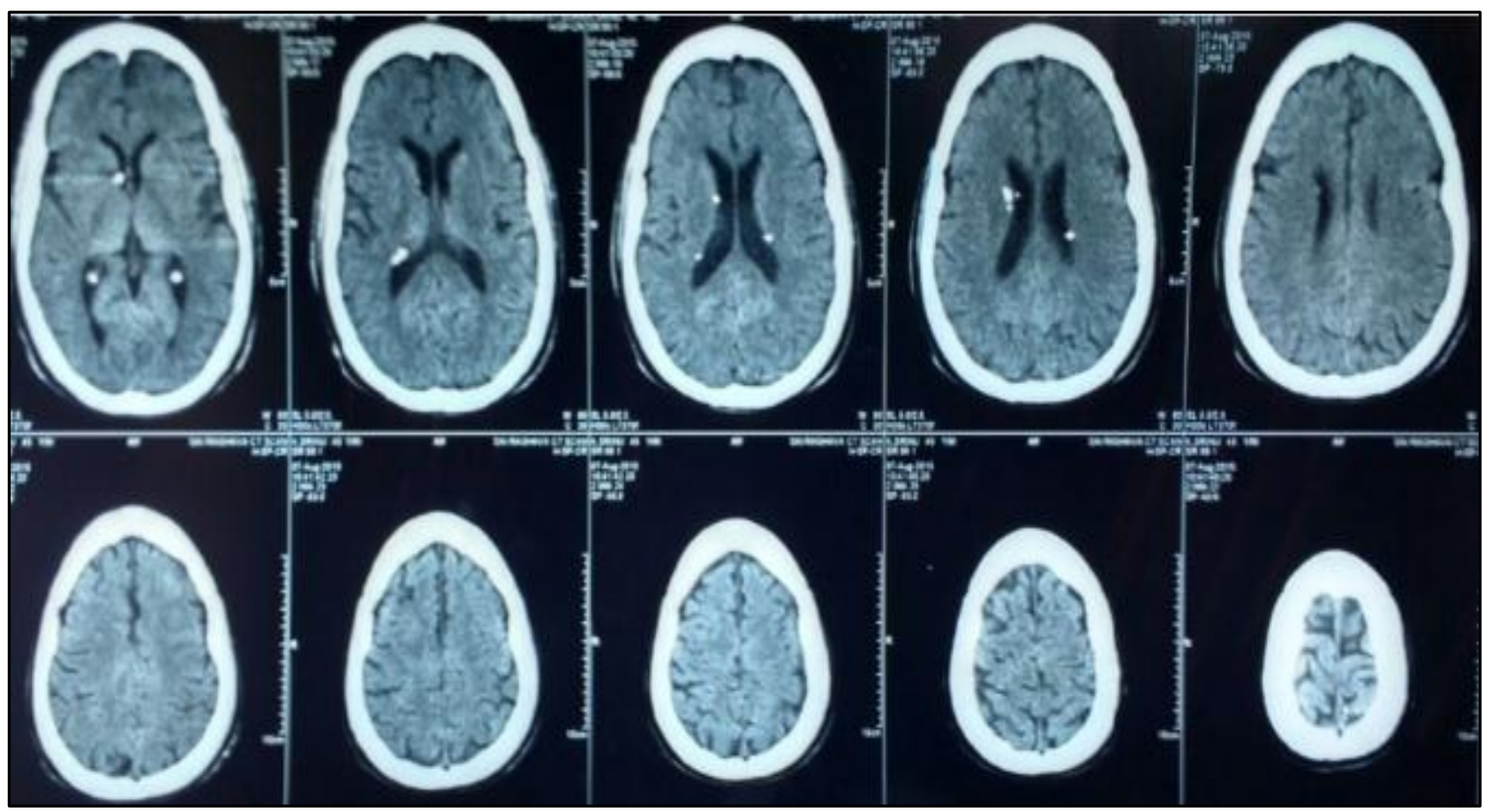

Fig. 7: CT scan brain showing multiple calcifications seen in both Subependymal and Peri-ventricular regions 


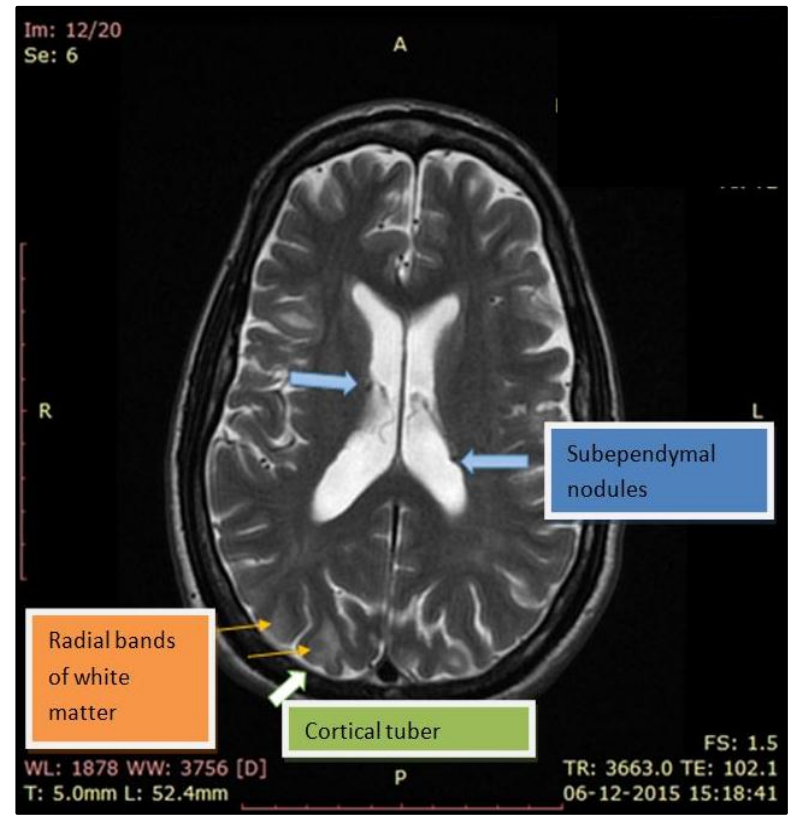

Fig. 8

\section{DISCUSSION}

Tuberous sclerosis was first described in 1880 by Bourneville (A French physician). The neurocutaneous syndrome is named for the firm whitish tuber like nodules arising from the cerebral convolutions. The most frequently involved organs are brain, kidneys, Lungs, heart, skin and skeleton.

\section{CNS}

Classical CNS involvement includes cortical tubers, subependymal nodules, subependymal giant cell astrocytomas and benign white matter lesions. Tubers are benign lesions composed of dysmorphic neurons occupying a cortical or subcortical location. They are detected by abnormal signal on MR due to distortion of affected gyrus. Cortical tubers may calcify. By age of $10,50 \%$ of patients have calcified cortical tubers. Subependymal nodules are found in nearly $95 \%$ of patients with tuberous sclerosis. ${ }^{3}$ the commonest site is near the caudate nucleus along the striothalamic groove of the lateral ventricle.

NCCT delineates periventricular and parenchymal calcification very well. Subependymal giant cell astrocytomas are histologically benign tumors located near the foramen of Monro. They are frequently calcified and associated obstructive hydrocephalous is a common symptom on presentation of the patient. ${ }^{4}$

\section{Kidney}

Renal lesions in tuberous sclerosis commonly consist of simple renal cyst and angiomyolipoma. Angiomyolipomas are benign in nature. They may arise in renal cortex or medulla. They are usually multiple and bi-lateral and are present in 50\% to $60 \%$ of the patients with tuberous sclerosis. ${ }^{5}$ On sonography classically angiomyolipomas are echogenic relative to renal parenchyma. If vascular element predominates, they may be hypoechoic.

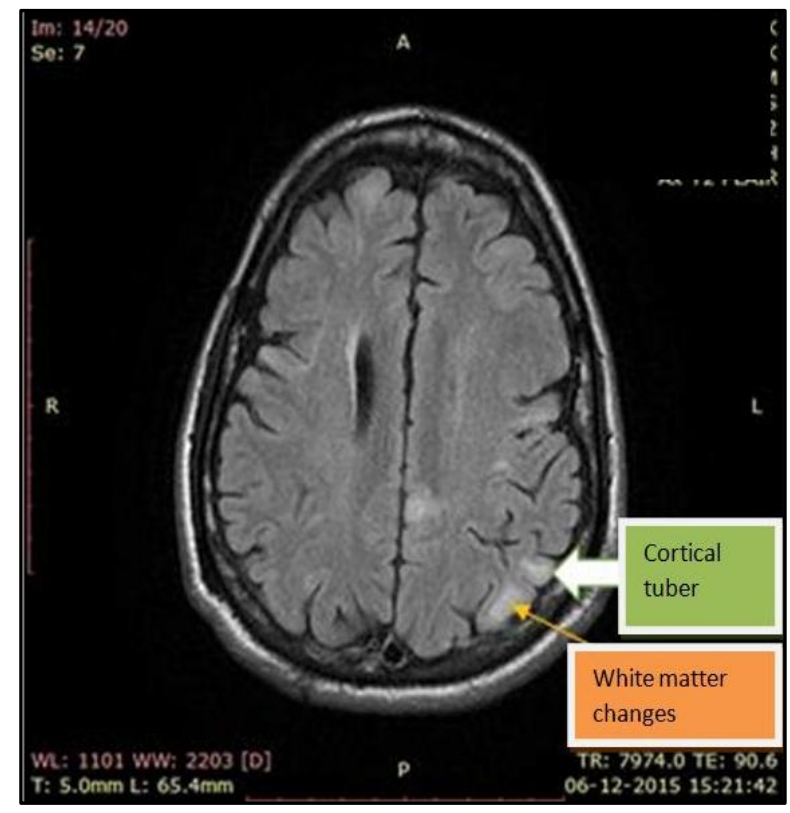

Fig. 9

Skin

Cutaneous lesions are present in $96 \%$ of the patients. These include facial angiofibromas (Adenoma sebaceum), subungual fibromas, Shagreen patches and café-au-lait spots.

In general the disease advances so slowly over a period of time, but in severe cases $30 \%$ of patients die before the age of 5 years and $50-75 \%$ of patients die when they attain adult age. Everolimus was approved for the treatment of subependymal giant cell astrocytomas. Dermabrasion and laser treatment may be useful for treatment of skin lesions.

\section{CONCLUSION}

Tuberous sclerosis is a genetic disorder with varied manifestations like angiofibromas distributed in a characteristic "butterfly" pattern on the face and forehead. The most important neurological problems are mental retardation, seizures and autism and learning difficulties. Treatment of seizures and genetic counselling, learning skills and education should be advocated in these patients.

\section{REFERENCES}

1. Shobha Bijjargi, Ashwini Rani SR, Veerendra S Patil, et al. Tuberous Sclerosis Complex Syndrome- A rare case report; Journal of Medical Education and Research; 2013;3(1);57-63.

2. TS Cheng. Tuberous sclerosis complex; an update review article, Hong Kong J Dermatol; Venereol 2012;20:61-67.

3. Braffman BH, Bilaniuk LT, Naidich TP, et al. MR imaging of tuberous sclerosis: pathogenesis of this phakomatosis. Use of gadopentetate dimeglumine and literature review. Radiology 1992; 183:227-238.

4. Kingsley D, Kendall B, Fitz C. Tuberous sclerosis: a clinic radiological evaluation of 110 cases with particular reference to atypical presentation. Neuroradio 1986; 28:171-190.

5. Stillwell TJ, Gomez MR, Kelalis PP. Renal lesions in tuberous sclerosis. J Urol 1987;137:477-481. 